\title{
Counting Handovers in a Cellular Mobile Communication Network: Equilibrium Renewal Process Approach
}

\author{
Ramón M. Rodríguez-Dagnino ${ }^{1}$ and Hideaki Takagi ${ }^{2}$
}

\begin{abstract}
Knowing the number of handovers that a user makes during a call session is particularly important in wireless cellular mobile communication networks in order to make appropriate dimensioning of virtual circuits for wireless cells. In this paper, we study the probability distributions and statistical moments for the number of handovers per call for a variety of combinations of the call holding time (CHT) and cell residence time (CRT) distributions. Based on the formulation in terms of equilibrium renewal processes, we obtain analytical expressions for the probability mass functions and moments of the handover number distribution. Numerical examples are provided that show a heavy-tail in the handover number distribution when the CHT has heavy tail.
\end{abstract}

Keywords: Cellular mobile communication networks; multimedia service; handover number; call holding time; cell residence time; renewal theory; equilibrium renewal process

\section{Introduction}

The evolution of wireless cellular mobile communication systems to provide multimedia services has motivated the study of new network performance issues. In multimedia networks it is expected to provide various services such as the traditional voice calls, facsimile, internet, data, still images, videotelephony, videoconference, and real-time video. In spite of the call holding time (CHT) and cell residence time (CRT) measurements reported in the literature for some of these services, we believe that most of them have not been fully characterized in the cellular multi-service environment in which the session for each service may present a different behavioral pattern. A large variety of requirements of different services would demand efficient sharing of communication channels, thus the characterization of the handover process arises as an important problem [11, 13]. The handover traffic is a complex function of many factors such as the size of a wireless cell, user's mobility pattern, radio channel environment, the call duration, etc. However, it has a direct impact on the signaling traffic, the call admission policy for new users, and also the quality of service (QoS) for the admitted users. For instance, the service disruption in the handover process may significantly degrade the specified QoS of timeconstrained services such as real-time audio and video. Hence the study of the handover process is a fundamental issue in the design of multimedia wireless cellular networks.

${ }^{1}$ Monterrey Institute of Technology (ITESM), Centro de Electrónica y Telecomunicaciones, Sucursal de correos "J", C.P. 64849, Monterrey, N.L., México. phone: +52-8358-2000 ext. 5029, fax: +52-83597211, e-mail: rmrodrig@campus.mty.itesm.mx

2 Institute of Policy and Planning Sciences, University of Tsukuba, Tsukuba-shi, Ibaraki 305-8573 Japan. phone: +81-298-53-5003, fax: +81-298-55-3849, e-mail: takagi@shako.sk.tsukuba.ac.jp 
There are many studies dealing with the handover statistics for voice calls. However the handover behavior of the new services is still in its early stage of investigation. A major difference is that the CHT cannot be assumed as an exponentially distributed random variable in multimedia services. The CRT is not exponentially distributed either. In this paper, we focus on the number of handovers during a call. Our approach is based on renewal theory arguments (see below), as it has been initiated in [15]. We obtain analytical expressions for the probability distribution and the statistical moments of the handover number for a variety of combinations of the CHT and CRT distributions. In particular, we present explicit results in the following cases: (a) a mixture of exponential or Erlang distributions for the CHT and a general distribution for the CRT, (b) an exponential distribution for the CRT and a general distribution for CHT (including gamma, lognormal, inverse Gaussian, and Pareto distributions), and (c) 2-Erlang distributions for both CHT and CRT. An important parameter to characterize the CHT is the coefficient of variation, denoted by $C_{V}$. By an exponentially distributed CHT we can model the call with $C_{V}=1$ as for traditional voice calls. A mixture of exponential distributions for the CHT allows us to model the heavy-tail traffic with $C_{V}>1$. The Erlang distributed CHT is a good model when $C_{V}<1$, and a mixture of Erlang distributions offers even a richer class for the CHT.

Nanda [14] uses a different methodology to solve this problem for the special case of exponentially distributed CRT and CHT. The same case has been extensively studied by Lin [10]. Recently, similar results have been obtained by Fang et al. [5, 6] by following another approach. They have obtained solutions as a set of recursive equations depending of the $k$ parameter in the $k$-Erlang distribution for the CHT. However, they observe that the recursive equations for obtaining the probability of call completion become much more involved as $k$ increases [5, p.1572]. In fact, they are not finding a general explicit solution to the $k$-Erlang or Pareto CHT, as we will do in this paper.

Let $N(t)$ be the number of renewals in a fixed time interval $[0, t]$, and the interrenewal times occur according to a sequence of random variables $\left\{X_{1}, X_{2}, \ldots, X_{i}, \ldots\right\}$, where $X_{1}$ is started at time 0 . This represents the sequence of CRTs that a mobile user experiences during a call such that $X_{i}$ is the CRT in the $i$ th cell $(i=1,2, \ldots)$. Now, let $T$ be a random variable representing a CHT; throughout the paper we do not take into account the forced termination of calls due to the blocking of handover process. Let us also assume that $T$ is independent of $\left\{X_{1}, X_{2}, \ldots, X_{i}, \ldots\right\}$. Hence $N(T)$ is a random variable which represents the number of renewals (handovers) in a random interval $[0, T]$ (a CHT). The problem of finding the probability distribution of $N(T)$ has been solved in several specific cases by Cox in his monograph [3, sec. 3.4] under the title "The number of renewals in a random time." Most of the results presented by Cox are based on the ordinary renewal process, i.e., all the random variables $X_{i}, i=1,2, \ldots$ come from the same distribution. However, a common situation in cellular networks is that a mobile user begins his call somewhere inside a cell. Thus it is more appropriate to consider the case in which only $X_{i}, i=2,3, \ldots$ come from the same distribution as a random variable $X$ while $X_{1}$ is the residual life of $X$, as noted by Lin [12]. This renewal process has also been studied by Cox in a different context, called the equilibrium renewal process in his monograph [3, sec. 2.2]. We denote by $f_{X}(x)$ the probability density function $(p d f)$ of $X$, and by $f_{X}^{*}(s):=\int_{0}^{\infty} e^{-s x} f_{X}(x) d x$ its Laplace transform. Similarly, the $p d f$ and its 


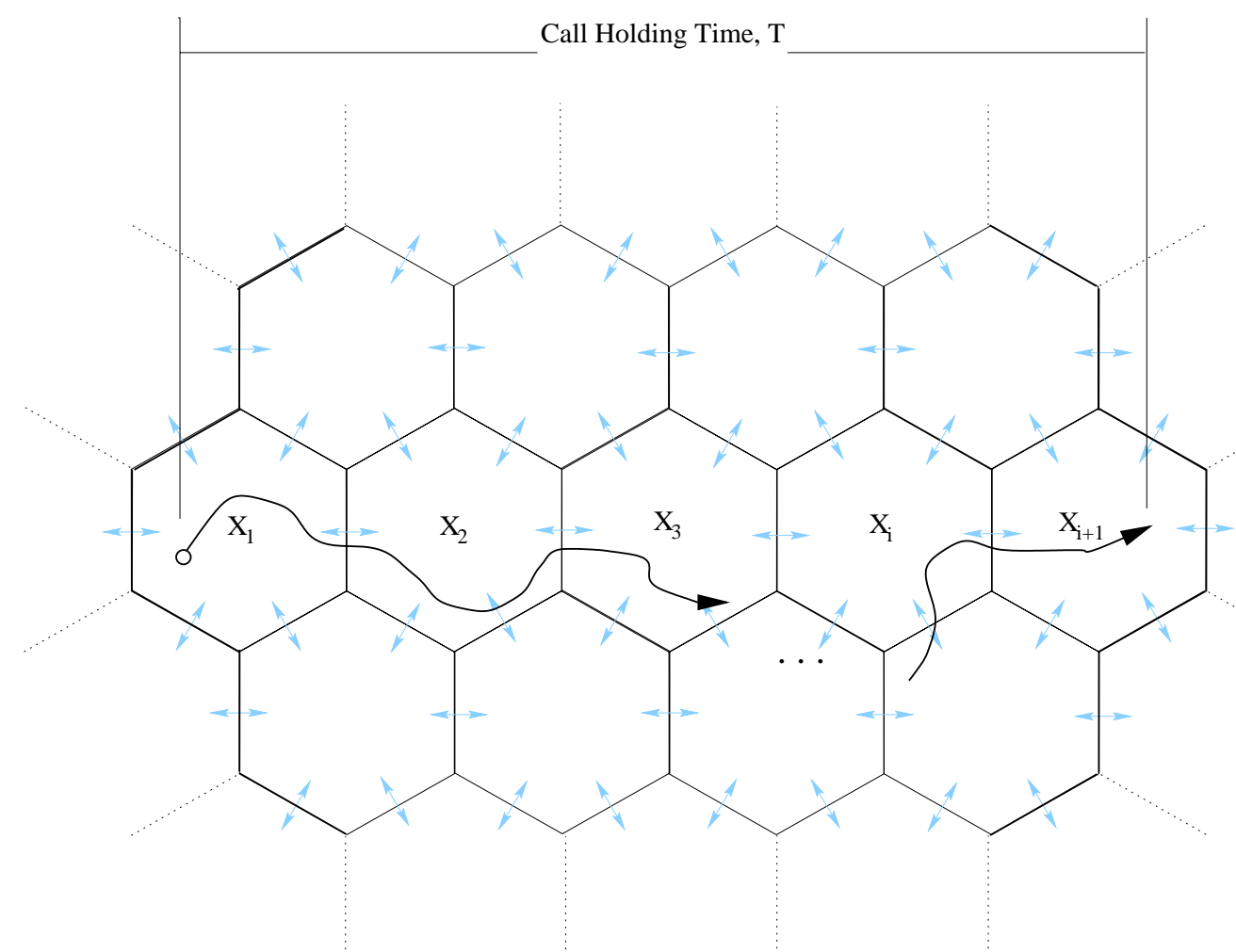

Figure 1: Mobility of a user in a cellular network. The random variable $X_{i}$ represents the CRT in the $i$ th cell $(i=1,2, \ldots)$, and $T$ represents the CHT.

Laplace transform of $T$ are denoted by $f_{T}(t)$ and $f_{T}^{*}(s)$, respectively.

Most of the results in this paper will be expressed as a function of the mobility ratio parameter

$$
\rho:=\frac{\text { Expected value of CHT }}{\text { Expected value of CRT }}=\frac{E[T]}{E[X]},
$$

which represents the average number of handovers a mobile user makes per call. See Figure 1 for the diagram of a CHT and CRTs associated with a mobile user in a cellular network.

The remainder of this paper is organized as follows. In Section 2 we present the basic methodology to calculate the probability generating function $(p g f)$ for $N(T)$ for CHT with Erlang distribution and for the CRT with any distribution. In Section 3, we consider special cases in which the CHT is exponentially distributed. We derive simple expressions for the probability mass function $(p m f) P[N(T)=j]$ as well as the binomial moments $E\left[\left(\begin{array}{c}N(T) \\ \ell\end{array}\right)\right]$ of $N(T)$ for the exponentially and gamma distributed CRT. In Section 4, we extend the method of Section 2 to the CHT with a mixture of several Erlang distributions, and present closed-form expressions for the pmf and the binomial moments of $N(T)$ when the CRT has exponential distribution and generalized gamma distribution. This richer class for the CHT allows us to model distributions with $C_{V} \leq 1$ or $C_{V}>1$ for the CHT. In Section 5 we turn to the case in which the CRT is exponentially distributed while CHT has arbitrary distribution. In this case, the $\operatorname{pmf} P[N(T)=j]$ 
can be expressed in terms of $f_{T}^{*}(s)$ and the moments of $N(T)$ in terms of those of $T$. In particular, we discuss the gamma, a mixture of gammas, lognormal, inverse Gaussian, and Pareto distributed CHT. We compare the resulting $p m f$ 's in these cases numerically, and find the heavy tail in $P[N(T)=j]$ for the Pareto CHT. The case of an Erlang distribution for the CHT and another Erlang distribution for the CRT seems to be cumbersome. In Section 6, we solve the case of 2-Erlang $(\lambda)$ distribution for the CHT and 2-Erlang $(\mu)$ distribution for the CRT, and evaluate the mean and variance of $N(T)$. In Section 7, we derive a general formula for the variance of $N(T)$ that is valid in the framework of the equilibrium renewal process. Finally, concluding remarks are made in Section 8.

\section{The $p g f$ Method for Erlang CHT}

Let $G_{N(T)}(z)$ be the $p g f$ for $N(T)$, the number of handovers in a random interval $[0, T]$. It is given by

$$
G_{N(T)}(z)=\int_{t=0}^{\infty} G_{N(t)}(t, z) f_{T}(t) d t
$$

where $f_{T}(t)$ is the $p d f$ of the random variable $T$, and

$$
G_{N(t)}(t, z):=\mathbf{E}\left[z^{N(t)}\right]=\sum_{j=0}^{\infty} P[N(T)=j \mid T=t] z^{j}
$$

is the $p g f$ of $N(t)$, the number of handovers in a fixed interval $[0, t]$. Once $G_{N(T)}(z)$ is obtained, the $p m f$ of $N(T)$ is given by

$$
P[N(T)=j]=\left.\frac{1}{j !} \frac{d^{j}}{d z^{j}} G_{N(T)}(z)\right|_{z=0} ; \quad j=0,1,2, \ldots .
$$

The $\ell$ th binomial moment of $N(T)$ is given by

$$
\mathbf{E}\left[\left(\begin{array}{c}
N(T) \\
\ell
\end{array}\right)\right]=\left.\frac{1}{\ell !} \frac{d^{\ell}}{d z^{\ell}} G_{N(T)}(z)\right|_{z=1} ; \quad \ell=0,1,2, \ldots
$$

By taking the derivative with respect to $z$ at both sides of (2), and evaluating at $z=1$ we find the following expression for the mean of the number of handovers:

$$
\mathbf{E}[N(T)]=\int_{t=0}^{\infty} \mathbf{E}[N(T) \mid T=t] f_{T}(t) d t .
$$

As shown by Cox [3, p.46, eq.(3)], it holds for the equilibrium renewal process that

$$
\mathbf{E}[N(T) \mid T=t]=\frac{t}{\mathbf{E}[X]}
$$

for any distribution $f_{X}(x)$ having $\mathbf{E}[X]$. Hence, we obtain

$$
\mathbf{E}[N(T)]=\int_{t=0}^{\infty} \frac{t}{\mathbf{E}[X]} f_{T}(t) d t=\frac{\mathbf{E}[T]}{\mathbf{E}[X]}=\rho .
$$


This general result has been obtained by a rather different argument by Nanda [14]. However, we remark that it is valid only for the equilibrium renewal process and for the ordinary renewal Poisson process.

Let us consider a special case in which the CHT can be fitted by a $k$-stage Erlang $p d f$, say

$$
f_{T}(t)=\frac{\lambda^{k} t^{k-1} e^{-\lambda t}}{(k-1) !} ; \quad t \geq 0
$$

with mean $\mathbf{E}[T]=k / \lambda$, so $\rho=k /(\lambda \mathbf{E}[X])$. In this case the relation between $G_{N(T)}(z)$ and $G_{N(t)}(t, z)$ has been solved by Cox [3, p.43, eq.(4)] as

$$
G_{N(T)}(z)=\left.\frac{\lambda^{k}}{(k-1) !}\left(-\frac{\partial}{\partial s}\right)^{k-1}\left\{G_{N(t)}^{*}(s, z)\right\}\right|_{s=\lambda}
$$

where $G_{N(t)}^{*}(s, z)$ is the Laplace transform of $G_{N(t)}(t, z)$ defined by

$$
G_{N(t)}^{*}(s, z):=\int_{t=0}^{\infty} e^{-s t} G_{N(t)}(t, z) d t .
$$

For the equilibrium renewal process, we have [3, p.38, eq.(6)]

$$
G_{N(t)}^{*}(s, z)=\frac{1}{s}+\frac{\left[1-f_{X}^{*}(s)\right](z-1)}{\mathbf{E}[X] s^{2}\left[1-f_{X}^{*}(s) z\right]},
$$

where $f_{X}^{*}(s)$ is the Laplace transform of the $p d f f_{X}(x)$ for the CRT $X$. Hence we get

$$
\begin{aligned}
G_{N(T)}(z) & =\left.\frac{\lambda^{k}}{(k-1) !}\left(-\frac{\partial}{\partial s}\right)^{k-1}\left\{\frac{1}{s}+\frac{\left[1-f_{X}^{*}(s)\right](z-1)}{\mathbf{E}[X] s^{2}\left[1-f_{X}^{*}(s) z\right]}\right\}\right|_{s=\lambda} \\
& =1+\left.\frac{\lambda^{k}(z-1)}{(k-1) ! \mathbf{E}[X]}\left(-\frac{\partial}{\partial s}\right)^{k-1} \frac{1-f_{X}^{*}(s)}{s^{2}\left[1-f_{X}^{*}(s) z\right]}\right|_{s=\lambda}
\end{aligned}
$$

From this we can express the $p m f$ of $N(T)$ as

$$
P[N(T)=j]=\left\{\begin{array}{ll}
1-\left.\frac{\lambda^{k}}{(k-1) ! \mathbf{E}[X]}\left(-\frac{\partial}{\partial s}\right)^{k-1} \frac{1-f_{X}^{*}(s)}{s^{2}}\right|_{s=\lambda} & ; j=0 \\
\left.\frac{\lambda^{k}}{(k-1) ! \mathbf{E}[X]}\left(-\frac{\partial}{\partial s}\right)^{k-1}\left[\frac{1-f_{X}^{*}(s)}{s}\right]^{2}\left[f_{X}^{*}(s)\right]^{j-1}\right|_{s=\lambda} \quad ; j=1,2, \ldots
\end{array} .\right.
$$

We can also express the $\ell$ th binomial moment of $N(T)$ as

$$
\mathbf{E}\left[\left(\begin{array}{c}
N(T) \\
\ell
\end{array}\right)\right]=\left.\frac{\lambda^{k}}{(k-1) ! \mathbf{E}[X]}\left(-\frac{\partial}{\partial s}\right)^{k-1} \frac{1}{s^{2}}\left[\frac{f_{X}^{*}(s)}{1-f_{X}^{*}(s)}\right]^{\ell-1}\right|_{s=\lambda} \quad \ell=1,2, \ldots .
$$

Thus we confirm that

$$
\mathbf{E}[N(T)]=\left.\frac{\lambda^{k}}{(k-1) ! \mathbf{E}[X]}\left(-\frac{\partial}{\partial s}\right)^{k-1} \frac{1}{s^{2}}\right|_{s=\lambda}=\frac{k}{\lambda \mathbf{E}[X]}=\rho
$$

regardless of the distributional form of $f_{X}(x)$. 


\section{Exponentially Distributed CHT and General CRT}

If we take $k=1$ in (9), we obtain an exponential $p d f$ for the random variable $T$ :

$$
f_{T}(t)=\lambda e^{-\lambda t} ; \quad t \geq 0
$$

with mean $\mathbf{E}[T]=1 / \lambda$. In this case, the pgf of $N(T)$ can be readily derived from (13) with $k=1$ as

$$
G_{N(T)}(z)=\left.\lambda\left\{G_{N(t)}^{*}(s, z)\right\}\right|_{s=\lambda}=1+\frac{\rho\left[1-f_{X}^{*}(\lambda)\right](z-1)}{1-f_{X}^{*}(\lambda) z},
$$

where $\rho=1 /(\lambda \mathbf{E}[X])$. The $j$ th derivative of this $p g f$ is given by

$$
\frac{d^{j}}{d z^{j}} G_{N(T)}(z)=\frac{j ! \rho\left[1-f_{X}^{*}(\lambda)\right]^{2}\left[f_{X}^{*}(\lambda)\right]^{j-1}}{\left[1-f_{X}^{*}(\lambda) z\right]^{j+1}} ; \quad j=1,2, \ldots
$$

Substituting (19) into (4), we obtain the pmf of $N(T)$ as

$$
P[N(T)=j]=\left\{\begin{array}{ll}
1-\rho\left[1-f_{X}^{*}(\lambda)\right] & ; j=0 \\
\rho\left[1-f_{X}^{*}(\lambda)\right]^{2}\left[f_{X}^{*}(\lambda)\right]^{j-1} & ; j=1,2, \ldots
\end{array},\right.
$$

which has also been obtained in [13, eq.(6)] by a different method.

Substituting (19) into (5), we obtain the $\ell$ th binomial moment of $N(T)$ as

$$
\mathbf{E}\left[\left(\begin{array}{c}
N(T) \\
\ell
\end{array}\right)\right]=\rho\left[\frac{f_{X}^{*}(\lambda)}{1-f_{X}^{*}(\lambda)}\right]^{\ell-1} ; \quad \ell=1,2, \ldots,
$$

from which the variance of $N(T)$ is given by

$$
\operatorname{Var}[N(T)]=\frac{2 \rho f_{X}^{*}(\lambda)}{1-f_{X}^{*}(\lambda)}+\rho(1-\rho)
$$

\subsection{Exponentially Distributed CRT}

Now, let us assume that the CRT is exponentially distributed as

$$
f_{X}(x)=\mu e^{-\mu x} ; \quad x \geq 0
$$

with mean $\mathbf{E}[X]=1 / \mu$, then $\rho=\mu / \lambda$. The Laplace transform of $f_{X}(x)$ is given by

$$
f_{X}^{*}(s):=\int_{x=0}^{\infty} e^{-s x} f_{X}(x) d x=\frac{\mu}{s+\mu} .
$$

Hence the pmf in (20) simplifies to

$$
P[N(T)=j]=\left(\frac{1}{1+\rho}\right)\left(\frac{\rho}{1+\rho}\right)^{j} ; \quad j=0,1,2, \ldots,
$$


which is a geometric distribution as shown in [13, eq.(8)]. Since both CHT and CRT have memoryless $p d f$, it is not surprising that the number of handovers during a call also has a (discrete) memoryless pmf, which can therefore only be geometric distribution.

The $\ell$ th binomial moment is simply given by

$$
\mathbf{E}\left[\left(\begin{array}{c}
N(T) \\
\ell
\end{array}\right)\right]=\rho^{\ell} ; \quad \ell=0,1,2, \ldots .
$$

Hence, the variance is given by

$$
\operatorname{Var}[N(T)]=\rho(1+\rho)
$$

\subsection{Gamma Distributed CRT}

In some cases, the CRT may be well-fitted by a gamma distribution, say

$$
f_{X}(x)=\frac{\mu(\mu x)^{\alpha-1}}{\Gamma(\alpha)} e^{-\mu x} ; \quad x \geq 0
$$

with mean $\mathbf{E}[X]=\alpha / \mu$, so $\rho=\mu /(\lambda \alpha)$. Here $\Gamma(\alpha):=\int_{0}^{\infty} u^{\alpha-1} e^{-u} d u, \alpha>0$, is the gamma function. The Laplace transform of $f_{X}(x)$ is given by

$$
f_{X}^{*}(s)=\left(\frac{\mu}{s+\mu}\right)^{\alpha} .
$$

In this case we get the $p m f$ of $N(T)$ as

$$
P[N(T)=j]=\left\{\begin{array}{ll}
1-\rho\left[1-\left(\frac{\alpha \rho}{1+\alpha \rho}\right)^{\alpha}\right] & ; j=0 \\
\rho\left[1-\left(\frac{\alpha \rho}{1+\alpha \rho}\right)^{\alpha}\right]^{2}\left(\frac{\alpha \rho}{1+\alpha \rho}\right)^{\alpha(j-1)} & ; j=1,2, \ldots
\end{array} .\right.
$$

The $\ell$ th binomial moment of $N(T)$ is given by

$$
\mathbf{E}\left[\left(\begin{array}{c}
N(T) \\
\ell
\end{array}\right)\right]=\rho\left[\frac{1}{\left(1+\frac{1}{\alpha \rho}\right)^{\alpha}-1}\right]^{\ell-1} ; \quad \ell=1,2, \ldots,
$$

which yields the variance

$$
\operatorname{Var}[N(T)]=\frac{2 \rho}{\left(1+\frac{1}{\alpha \rho}\right)^{\alpha}-1}+\rho(1-\rho) .
$$

\section{The $p g f$ Method for Mixed-Erlang CHT}

Let us assume that the call holding time $T$ is well modeled by a mixture of $M$ Erlang distributions, say

$$
f_{T}(t)=\sum_{i=1}^{M} p_{i} \frac{\lambda_{i}^{k_{i}} t^{k_{i}-1} e^{-\lambda_{i} t}}{\left(k_{i}-1\right) !} ; \quad t \geq 0
$$


where $\sum_{i=1}^{M} p_{i}=1$. Then the $p g f$ of $N(T)$ is given by

$$
G_{N(T)}(z)=\left.\sum_{i=1}^{M} p_{i} \frac{\lambda_{i}^{k_{i}}}{\left(k_{i}-1\right) !}\left(-\frac{\partial}{\partial s}\right)^{k_{i}-1}\left\{G_{N(t)}^{*}(s, z)\right\}\right|_{s=\lambda_{i}},
$$

where $G_{N(t)}^{*}(s, z)$ is given by (12). Thus we can obtain the expressions for the pmf and moments of $N(T)$ similar to (14) through (16).

Some special cases are studied below.

\subsection{Mixture of Exponential Distributions for the CHT}

If we consider a mixture of exponential distributions for the CHT, or $k_{i}=1$ for all $i$ in (34), we obtain

$$
G_{N(T)}(z)=1+\sum_{i=1}^{M} p_{i} \frac{\left[1-f_{X}^{*}\left(\lambda_{i}\right)\right](z-1)}{\lambda_{i} \mathbf{E}[X]\left[1-f_{X}^{*}\left(\lambda_{i}\right) z\right]},
$$

where $T=\sum_{i=1}^{M} p_{i} T_{i}$ is the mixture of $M$ exponentially distributed random variables $T_{i}$ with mean $\mathbf{E}\left[T_{i}\right]=1 / \lambda_{i}$. The $j$ th derivative of this $p g f$ is given by

$$
\frac{d^{j}}{d z^{j}} G_{N(T)}(z)=j ! \sum_{i=1}^{M} p_{i} \frac{\rho_{i}\left[1-f_{X}^{*}\left(\lambda_{i}\right)\right]^{2}\left[f_{X}^{*}\left(\lambda_{i}\right)\right]^{j-1}}{\left[1-f_{X}^{*}\left(\lambda_{i}\right) z\right]^{j+1}} ; \quad j=1,2, \ldots,
$$

where we have defined $\rho_{i}:=\mathbf{E}\left[T_{i}\right] / \mathbf{E}[X]=1 /\left(\lambda_{i} \mathbf{E}[X]\right)$.

Hence, the $p m f$ of $N(T)$ is given by

$$
P[N(T)=j]=\left\{\begin{array}{ll}
1-\sum_{i=1}^{M} p_{i} \rho_{i}\left[1-f_{X}^{*}\left(\lambda_{i}\right)\right] & ; j=0 \\
\sum_{i=1}^{M} p_{i} \rho_{i}\left[1-f_{X}^{*}\left(\lambda_{i}\right)\right]^{2}\left[f_{X}^{*}\left(\lambda_{i}\right)\right]^{j-1} & ; j=1,2, \ldots
\end{array} .\right.
$$

The th binomial moment of $N(T)$ is given by

$$
\mathbf{E}\left[\left(\begin{array}{c}
N(T) \\
\ell
\end{array}\right)\right]=\sum_{i=1}^{M} p_{i} \rho_{i}\left[\frac{f_{X}^{*}\left(\lambda_{i}\right)}{1-f_{X}^{*}\left(\lambda_{i}\right)}\right]^{\ell-1} ; \quad \ell=1,2, \ldots .
$$

Thus we have $\mathbf{E}[N(T)]=\sum_{i=1}^{M} p_{i} \rho_{i}=\rho$, and

$$
\operatorname{Var}[N(T)]=2 \sum_{i=1}^{M} p_{i} \frac{\rho_{i} f_{X}^{*}\left(\lambda_{i}\right)}{1-f_{X}^{*}\left(\lambda_{i}\right)}+\rho(1-\rho) .
$$

\subsection{Exponentially Distributed CRT}

If we assume in (37) that each CRT is exponentially distributed with mean $1 / \mu$, as in (24), the pmf for $N(T)$ is a mixture of geometric random variables as follows:

$$
P[N(T)=j]=\sum_{i=1}^{M} p_{i}\left(\frac{1}{1+\rho_{i}}\right)\left(\frac{\rho_{i}}{1+\rho_{i}}\right)^{j} ; \quad j=0,1,2, \ldots,
$$


where $\rho_{i}=\mu / \lambda_{i}$.

The $\ell$ th binomial moment of $N(T)$ is given by

$$
\mathbf{E}\left[\left(\begin{array}{c}
N(T) \\
\ell
\end{array}\right)\right]=\sum_{i=1}^{M} p_{i} \rho_{i}^{\ell} ; \quad \ell=0,1,2, \ldots .
$$

The variance is given by

$$
\operatorname{Var}[N(T)]=2 \sum_{i=1}^{M} p_{i} \rho_{i}^{2}+\rho(1-\rho)
$$

\subsection{Generalized Gamma Distributed CRT}

According to Zonoozi and Dassanayake [22], the CRT can be modeled as a generalized gamma distribution with the $p d f$

$$
f_{X}(x)=\frac{c x^{c \alpha-1}}{\Gamma(\alpha) b^{c \alpha}} e^{-(x / b)^{c}} ; \quad x \geq 0, \alpha, b, c>0 .
$$

The $p d f$ in (43) may not have a rational Laplace transform, but its $k$ th moment about the origin is given by

$$
\mathbf{E}\left[X^{k}\right]=\frac{b^{k} \Gamma(\alpha+k / c)}{\Gamma(\alpha)} ; \quad k=0,1,2, \ldots
$$

We can obtain several special cases of (43) by selecting the corresponding parameters. For instance, we can obtain the gamma distribution by choosing $c=1$ and $b=1 / \mu$, and from here the exponential distribution by letting $\alpha=1$.

We should remember that for a random variable $X$ with $p d f f_{X}(x)$ its Laplace transform $f_{X}^{*}(s)$ can be expanded in the moments of $X$ as follows:

$$
f_{X}^{*}(s)=\sum_{k=0}^{\infty}(-1)^{k} \frac{s^{k}}{k !} \mathbf{E}\left[X^{k}\right] .
$$

Hence, by using the moments in $(44)$, we can expand $f_{X}^{*}\left(\lambda_{i}\right)$ as

$$
f_{X}^{*}\left(\lambda_{i}\right)=1+\sum_{k=1}^{\infty}(-1)^{k} \frac{G_{k}}{k ! \rho_{i}^{k}}
$$

where

$$
G_{k}=\frac{\Gamma^{k-1}(\alpha) \Gamma(\alpha+k / c)}{\Gamma^{k}(\alpha+1 / c)} ; \quad k=1,2, \ldots
$$

and

$$
\rho_{i}:=\frac{\mathbf{E}\left[T_{i}\right]}{\mathbf{E}[X]}=\frac{\Gamma(\alpha)}{\lambda_{i} b \Gamma(\alpha+1 / c)} .
$$

Note that the dependence on the parameters $\lambda_{i}$ and $b$ is concentrated only in the relative mobility ratio $\rho_{i}$ given in (48). The expansion in (46) can be substituted into (37) and (38) to obtain the $p m f$ and the moments of $N(T)$, respectively. We also note that the series expansion for $f_{X}^{*}(s)$ in (45) is useful to obtain the pmf and the moments of $N(T)$ for those distributions of $X$ that do not have closed-form expression for the Laplace transform. 


\section{General CHT and Exponentially Distributed CRT}

We now turn to assume that the CRT $X$ is exponentially distributed as in (23). Since $\mathbf{E}[X]=1 / \mu$, we have

$$
\rho=\mu \mathbf{E}[T]
$$

In this case, the sequence of CRTs is called a Poisson renewal process. Thus the $p g f$ for $N(t)$, the number of renewals in $[0, t]$, is given by

$$
G_{N(t)}(t, z)=\sum_{n=0}^{\infty} \frac{(\mu t)^{n}}{n !} e^{-\mu t} z^{n}=e^{-\mu(1-z) t} .
$$

This is a well-known result [9, p.63, eq.(2.134)]. As a consequence of the memoryless property of the exponential distribution, this result is valid for both the ordinary and the equilibrium renewal processes.

It can be observed from (2) and (50) that for any distribution for the CHT we can find that

$$
G_{N(T)}(z)=\int_{t=0}^{\infty} e^{-\mu(1-z) t} f_{T}(t) d t=f_{T}^{*}[\mu(1-z)],
$$

where $f_{T}^{*}(s)$ is the Laplace transform of $f_{T}(t)$. The $j$ th derivative of this $p g f$ is given by

$$
\frac{d^{j}}{d z^{j}} G_{N(T)}(z)=\int_{t=0}^{\infty}(\mu t)^{j} e^{-\mu(1-z) t} f_{T}(t) d t ; \quad j=0,1,2, \ldots
$$

Substituting (52) into (4) we can obtain the following $p m f$ for $N(T)$

$$
P[N(T)=j]=\int_{t=0}^{\infty} \frac{(\mu t)^{j}}{j !} e^{-\mu t} f_{T}(t) d t=\frac{(-\mu)^{j}}{j !} f_{T}^{*(j)}(\mu) ; \quad j=0,1,2, \ldots,
$$

which is a Poisson mixture of the $p d f f_{T}(t)$.

It is also possible to obtain a general expression for the binomial moments of $N(T)$. Substituting (52) into (5), we obtain

$$
\mathbf{E}\left[\left(\begin{array}{c}
N(T) \\
\ell
\end{array}\right)\right]=\frac{\mu^{\ell}}{\ell !} \mathbf{E}\left[T^{\ell}\right] ; \quad \ell=1,2, \ldots .
$$

Thus we get

$$
\mathbf{E}[N(T)]=\mu \mathbf{E}[T]=\rho
$$

and

$$
\operatorname{Var}[N(T)]=\mu^{2} \mathbf{E}\left[T^{2}\right]+\rho(1-\rho) .
$$

For instance, if $f_{T}(t)=\lambda e^{-\lambda t}$, we have $\mathbf{E}\left[T^{\ell}\right]=\ell ! / \lambda^{\ell}$, and

$$
\mathbf{E}\left[\left(\begin{array}{c}
N(T) \\
\ell
\end{array}\right)\right]=\left(\frac{\mu}{\lambda}\right)^{\ell}=\rho^{\ell} ; \quad \ell=0,1,2, \ldots,
$$

which agrees with (26). 


\subsection{Gamma CHT}

Let us first assume $\operatorname{Gamma}(\alpha, \lambda)$ for the CHT, which is more general than the $k$ $\operatorname{Erlang}(\lambda)$ distribution, as

$$
f_{T}(t)=\frac{\lambda(\lambda t)^{\alpha-1}}{\Gamma(\alpha)} e^{-\lambda t} ; \quad t \geq 0
$$

with mean $\mathbf{E}[T]=\alpha / \lambda$, so $\rho=\alpha \mu / \lambda$. The Laplace transform of $f_{T}(t)$ is given by

$$
f_{T}^{*}(s):=\int_{t=0}^{\infty} e^{-s t} f_{T}(t) d t=\left(\frac{\lambda}{s+\lambda}\right)^{\alpha} .
$$

By substituting (58) into (53) we find

$$
P[N(T)=j]=\frac{\Gamma(\alpha+j)}{\Gamma(\alpha) j !}\left(\frac{\alpha}{\alpha+\rho}\right)^{\alpha}\left(\frac{\rho}{\alpha+\rho}\right)^{j} ; j=0,1,2, \ldots .
$$

This is the Pólya-Eggenberger $p m f$. The $\ell$ th binomial moment is given by

$$
\mathbf{E}\left[\left(\begin{array}{c}
N(T) \\
\ell
\end{array}\right)\right]=\frac{\Gamma(\alpha+\ell)}{\Gamma(\alpha) \ell !}\left(\frac{\mu}{\lambda}\right)^{\ell}=\frac{\Gamma(\alpha+\ell)}{\Gamma(\alpha) \ell !}\left(\frac{\rho}{\alpha}\right)^{\ell} ; \quad \ell=0,1,2, \ldots .
$$

The variance is given by

$$
\operatorname{Var}[N(T)]=\rho\left(1+\frac{\rho}{\alpha}\right)
$$

If $\alpha=k$ (a positive integer), it corresponds to a negative binomial $p m f$

$$
P[N(T)=j]=\left(\begin{array}{c}
k+j-1 \\
k-1
\end{array}\right)\left(\frac{k}{k+\rho}\right)^{k}\left(\frac{\rho}{k+\rho}\right)^{j} ; j=0,1,2, \ldots,
$$

where $\rho=k \mu / \lambda$. The special case $k=1$ reduces to $(25)$.

\subsection{Mixture of Gammas for CHT}

We next consider a general mixture of Gamma distributions for the CHT

$$
f_{T}(t)=\sum_{i=1}^{M} p_{i} \frac{\lambda_{i}\left(\lambda_{i} t\right)^{\alpha_{i}-1}}{\Gamma\left(\alpha_{i}\right)} e^{-\lambda_{i} t} ; \quad t \geq 0,
$$

where $\sum_{i=1}^{M} p_{i}=1$. This has the mean $E[T]=\sum_{i=1}^{M} p_{i} \alpha_{i} / \lambda_{i}$, so $\rho=\mu \sum_{i=1}^{M} p_{i} \alpha_{i} / \lambda_{i}$.

The $p m f$ of $N(T)$ is given by

$$
P[N(T)=j]=\sum_{i=1}^{M} p_{i} \frac{\Gamma\left(\alpha_{i}+j\right)}{\Gamma\left(\alpha_{i}\right) j !}\left(\frac{\alpha_{i}}{\alpha_{i}+\rho_{i}}\right)^{\alpha_{i}}\left(\frac{\rho_{i}}{\alpha_{i}+\rho_{i}}\right)^{j} ; \quad j=0,1,2, \ldots,
$$

where $\rho_{i}=\mu \alpha_{i} / \lambda_{i}$. The $\ell$ th binomial moment of $N(T)$ is given by

$$
\mathbf{E}\left[\left(\begin{array}{c}
N(T) \\
\ell
\end{array}\right)\right]=\sum_{i=1}^{M} p_{i} \frac{\Gamma\left(\alpha_{i}+\ell\right)}{\Gamma\left(\alpha_{i}\right) \ell !}\left(\frac{\rho_{i}}{\alpha_{i}}\right)^{\ell} ; \quad \ell=0,1,2, \ldots,
$$

which leads to the variance

$$
\operatorname{Var}[N(T)]=\sum_{i=1}^{M} p_{i} \rho_{i}^{2}\left(1+\frac{1}{\alpha_{i}}\right)+\rho(1-\rho) .
$$

A special case in which $\alpha_{i}=1$ for $i=1,2, \ldots, M$ reduces to the case in Section 4.2. 


\subsection{Lognormal CHT}

The lognormal distribution and a mixture thereof have been proposed as a model to capture the statistics of the CHT for several internet-related services, such as the circuit holding time in the dial-up access to an internet service provider (ISP), the number of message bytes in TCP/IP flows, and the e-mail message length [1].

The $p d f$ for the lognormal distribution of the CHT is given by

$$
f_{T}(t)=\frac{1}{\sqrt{2 \pi} \sigma t} \exp \left[-\frac{(\ln t-m)^{2}}{2 \sigma^{2}}\right] ; \quad t>0 .
$$

This can be obtained from the $Y \sim \operatorname{Gaussian}\left(m, \sigma^{2}\right)$ distribution by making the transformation $T=e^{Y}$. By substituting (68) into (53) and changing the parameter by $\phi:=\ln (\mu t)$, we obtain the Poisson-lognormal distribution

$$
P[N(T)=j]=\frac{1}{\sigma \sqrt{2 \pi} j !} \int_{\phi=-\infty}^{\infty} \exp \left[-e^{\phi}+j \phi-\frac{(\phi-\ln \mu-m)^{2}}{2 \sigma^{2}}\right] d \phi ; \quad j=0,1,2, \ldots .
$$

It is well-known that this does not have an explicit expression, and it has been tabulated in many places, e.g. in [20].

The $\ell$ th central moment for the lognormal distribution is given by

$$
\mathbf{E}\left[T^{\ell}\right]=e^{\ell m+\frac{1}{2} \ell^{2} \sigma^{2}} ; \quad \ell=0,1,2, \ldots
$$

Hence we get

$$
\mathbf{E}\left[\left(\begin{array}{c}
N(T) \\
\ell
\end{array}\right)\right]=\frac{\mu^{\ell}}{\ell !} e^{\ell m+\frac{1}{2} \ell^{2} \sigma^{2}} ; \quad \ell=0,1,2, \ldots .
$$

In particular, we have

$$
\mathbf{E}[N(T)]=\mu \mathbf{E}[T]=\mu e^{m+\frac{1}{2} \sigma^{2}}=\rho
$$

and

$$
\operatorname{Var}[N(T)]=\mu^{2} e^{2\left(m+\sigma^{2}\right)}+\rho(1-\rho)
$$

\subsection{Inverse Gaussian CHT}

The inverse Gaussian pdf has been proposed as an alternative to the lognormal distribution. It has an advantage that it is possible to find an explicit expression for the pmf of the Poisson-inverse Gaussian distribution which has behavior similar to the Poissonlognormal one. There are several related expressions to define the inverse Gaussian $p d f$, and the so-called canonical definition is as follows [8]:

$$
f_{T}(t)=\sqrt{\frac{\lambda}{2 \pi t^{3}}} \exp \left[-\frac{\lambda(t-m)^{2}}{2 m^{2} t}\right] ; \quad t>0, m, \lambda>0 .
$$

Note that $E[T]=m$ and that $\lambda$ is another parameter. 
Substituting (74) into (53), we obtain the following Poisson-inverse Gaussian $p m f$ for $N(T)$ :

$$
P[N(T)=j]=\frac{\mu^{j} e^{\lambda / m}}{j !}\left(\frac{2 \lambda}{\pi}\right)^{\frac{1}{2}}\left[\frac{\lambda}{2\left(\mu+\frac{\lambda}{2 m^{2}}\right)}\right]^{\frac{1}{2}\left(j-\frac{1}{2}\right)} K_{j-\frac{1}{2}}\left[\sqrt{2 \lambda\left(\mu+\frac{\lambda}{2 m^{2}}\right)}\right]
$$

for $j=0,1, \ldots$, where $K_{\nu}(z)$ is the modified Bessel function of second kind, or the Kelvin function, defined by

$$
K_{-\frac{1}{2}}(z)=\sqrt{\frac{\pi}{2 z}} e^{-z} \quad ; \quad K_{\nu}(z)=\frac{1}{2}\left(\frac{z}{2}\right)^{\nu} \int_{t=0}^{\infty} \frac{e^{-t-\frac{z^{2}}{4 t}}}{t^{\nu+1}} d t ; \quad|\arg z|<\frac{\pi}{4}, \nu>-\frac{1}{2} .
$$

The $p g f$ of $N(T)$ is given by

$$
G_{N(T)}(z)=\exp \left\{\frac{\lambda}{m}\left[1-\sqrt{1+\frac{2 m^{2}}{\lambda} \mu(1-z)}\right]\right\}
$$

From this $p g f$ we get $\mathbf{E}[N(T)]=m \mu=\rho$ and

$$
\operatorname{Var}[N(T)]=\rho\left(1+\frac{m}{\lambda} \rho\right)
$$

It seems that there are no simple expressions for a general moments of $N(T)$.

\subsection{Pareto CHT}

The Pareto $p d f$ has been proposed in several statistical data fitting for the www and http traffic which show heavy-tail behavior $[2,4]$. Let us consider the following Pareto $p d f$ for the CHT:

$$
f_{T}(t)=\frac{\alpha \beta^{\alpha}}{t^{\alpha+1}} ; \quad 1<\alpha<2, t \geq \beta>0 .
$$

This is a heavy-tail distribution with mean $\mathbf{E}[T]=\alpha \beta /(\alpha-1)$. It is well-known that the higher moments of this distribution do not exist. The tail is heavier as $\alpha$ is closer to unity. From (55), we have

$$
\rho=\frac{\mu \alpha \beta}{\alpha-1}
$$

The $p g f$ for $N(T)$ can be readily obtained from (51) as

$$
G_{N(T)}(z)=\alpha[\mu \beta(1-z)]^{\alpha} \int_{u=\mu \beta(1-z)}^{\infty} u^{-\alpha-1} e^{-u} d u .
$$

We get the pmf for $N(T)$ from (53) as

$$
P[N(T)=j]=\frac{\alpha(\mu \beta)^{\alpha}}{j !} \int_{u=\mu \beta}^{\infty} u^{-\alpha+j-1} e^{-u} d u ; \quad j=0,1,2, \ldots,
$$


which can be rewritten as

$$
P[N(T)=j]=\frac{\alpha(\mu \beta)^{\frac{\alpha+j-1}{2}}}{j !} e^{-\frac{\mu \beta}{2}} W_{\frac{j-\alpha-1}{2}, \frac{j-\alpha}{2}}(\mu \beta) ; \quad j=0,1,2, \ldots
$$

Here

$$
W_{\eta, \xi}(\phi):=\frac{\phi^{\eta} e^{-\phi / 2}}{\Gamma\left(\xi-\eta+\frac{1}{2}\right)} \int_{t=0}^{\infty} t^{\xi-\eta-\frac{1}{2}} e^{-t}\left(1+\frac{t}{\phi}\right)^{\xi+\eta-\frac{1}{2}} d t ; \quad \Re(\xi-\eta)>-\frac{1}{2},|\arg \phi|<\pi
$$

is the Whittaker's function [21, p.340], and we have used the formula [7, p.318]

$$
\int_{x=\xi}^{\infty} e^{-x} x^{-\nu} d x=\xi^{-\nu / 2} e^{-\xi / 2} W_{-\frac{\nu}{2}, \frac{1-\nu}{2}}(\xi) ; \quad \xi>0 .
$$

Since $\mu \beta=\rho(\alpha-1) / \alpha$, the pmf for $N(T)$ in (83) is a function of the mobility ratio $\rho$ and the parameter $\alpha$ of the Pareto distributed CHT. We also note that the variance for $N(T)$ is not defined, because the second moment for the Pareto $p d f$ does not exist.

\subsection{Numerical Comparison}

In Figure 2 we plot the $\operatorname{pmf} P[N(T)=j]$ for the mobility ratio $\rho=2$ for three Pareto CHT cases, $\alpha=1.1,1.5$, and 1.9, and also for the 3-Erlang and exponentially distributed CHT. We can observe that the pmf in the 3-Erlang case decays faster than the exponential case. However, all the Pareto cases have heavier tails, and the speed of decay in these cases depend on the parameter $\alpha$; the heavier is the tail in the CHT distribution ( $\alpha$ is closer to unity), the slower is the decay in the pmf $P[N(T)=j]$. In Figure 3, we can observe a similar behavior when $\rho$ is fixed at 20. In this situation, the decay is slower than the cases with $\rho=2$ shown in Figure 2.

It is interesting to compare the tails in the pmf $P[N(T)=j]$ for the Poissonlognormal, Poisson-inverse Gaussian, and Poisson-Pareto distributions for the CHT. In Figure 4, we have fixed the mean of all pmf's equal to 0.1 , and the variance equal to 0.2 for the first two distributions. For the Poisson-Pareto distribution, we have assumed the same mean and considered $\alpha=1.1,1.5$, and 1.9. We observe that the Poisson-lognormal has a heavier tail than the Poisson-Inverse Gaussian, and that all Poisson-Pareto cases have heavier tails than the Poisson-lognormal. As it has been discussed above, the Poisson-Pareto with $\alpha=1.1$ has the heaviest tail. A similar behavior can be observed in Figure 5, where we have considered the mean value of 6 for all $p m f$ 's, and the variance of 10 for the Poisson-lognormal and Poisson-inverse Gaussian distributions.

\section{2-Erlang CHT and 2-Erlang CRT}

We consider an interesting special case where the exponential distribution is not assumed either for the CHT or for the CRT. Specifically, let us assume the 2-Erlang $(\lambda)$ for CHT, i.e., $k=2$ in $(9)$, and the 2 -Erlang $(\mu)$ for CRT, i.e., $\alpha=2$ in (28). 


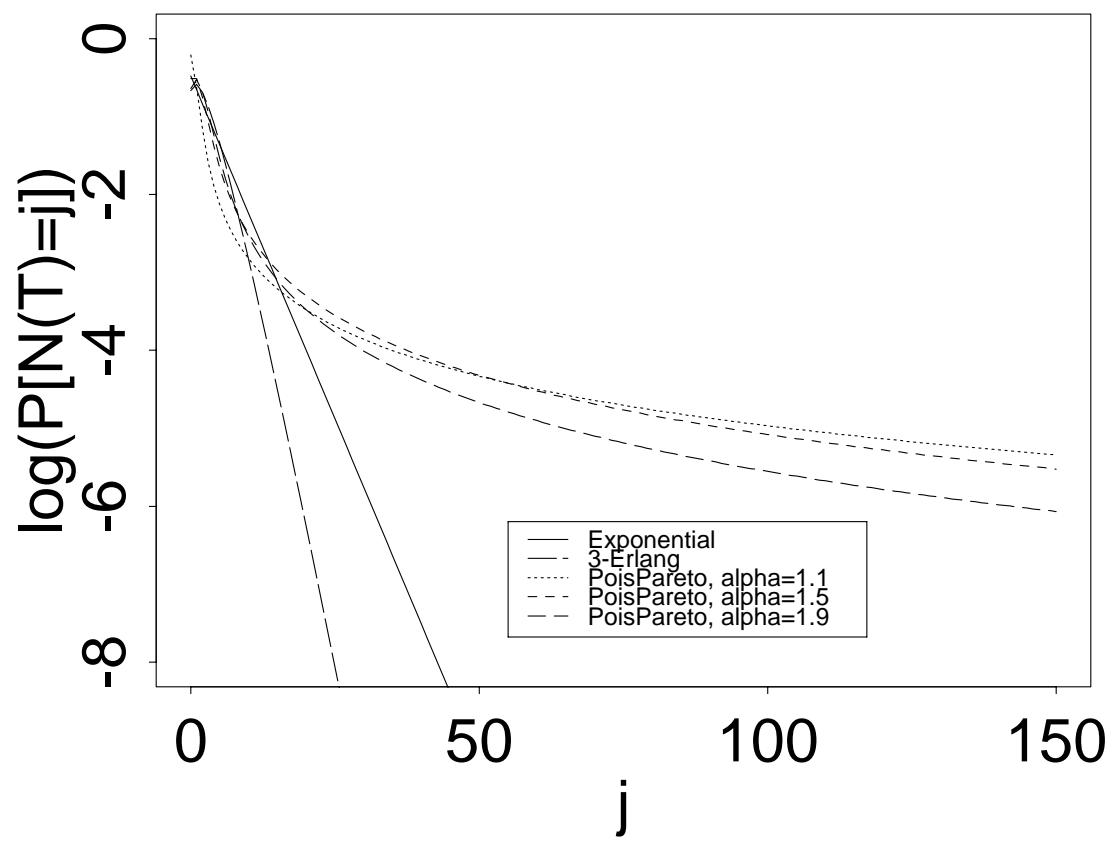

Figure 2: Logarithm of the pmf of the number of handovers, $\log _{10}(P[N(T)=j])$. We have fixed the mobility ratio as $\rho=2$, and we are comparing the following distributions for the CHT: Pareto with $\alpha=1.1,1.5$, and 1.9, 3-Erlang, and exponential. We assume the exponentially distributed CRT.

In this case we have $E[T]=2 / \lambda$ and $E[X]=2 / \mu$, and so $\rho=E[T] / E[X]=\mu / \lambda$. By following the same procedure as above, it can be shown that the $p g f$ of $N(T)$ is given by

$$
G_{N(T)}(z)=\pi \frac{(1-q)^{2}}{(1-q z)^{2}}+(1-\pi) \frac{(1-q)^{2} z}{(1-q z)^{2}}
$$

where

$$
\pi:=\frac{1+\rho}{1+2 \rho} \quad ; \quad q:=\left(\frac{\rho}{1+\rho}\right)^{2} .
$$

This corresponds to a mixture of two negative binomial $p m f \mathrm{~s}$ as

$$
P[N(T)=j]=\left\{\begin{array}{ll}
\pi P_{N B}(0,2, q) & ; j=0 \\
\pi P_{N B}(j, 2, q)+(1-\pi) P_{N B}(j-1,2, q) & ; j=1,2, \ldots
\end{array},\right.
$$

where

$$
P_{N B}(j, k, q):=\left(\begin{array}{c}
k+j-1 \\
k-1
\end{array}\right)(1-q)^{k} q^{j} ; \quad j=0,1,2, \ldots .
$$

The $\ell$ th binomial moment of $N(T)$ is given by

$$
\mathbf{E}\left[\left(\begin{array}{c}
N(T) \\
\ell
\end{array}\right)\right]=\pi \frac{(\ell+1) q^{\ell}}{(1-q)^{\ell}}+(1-\pi) \frac{(q+\ell) q^{\ell-1}}{(1-q)^{\ell}} ; \quad \ell=1,2, \ldots
$$




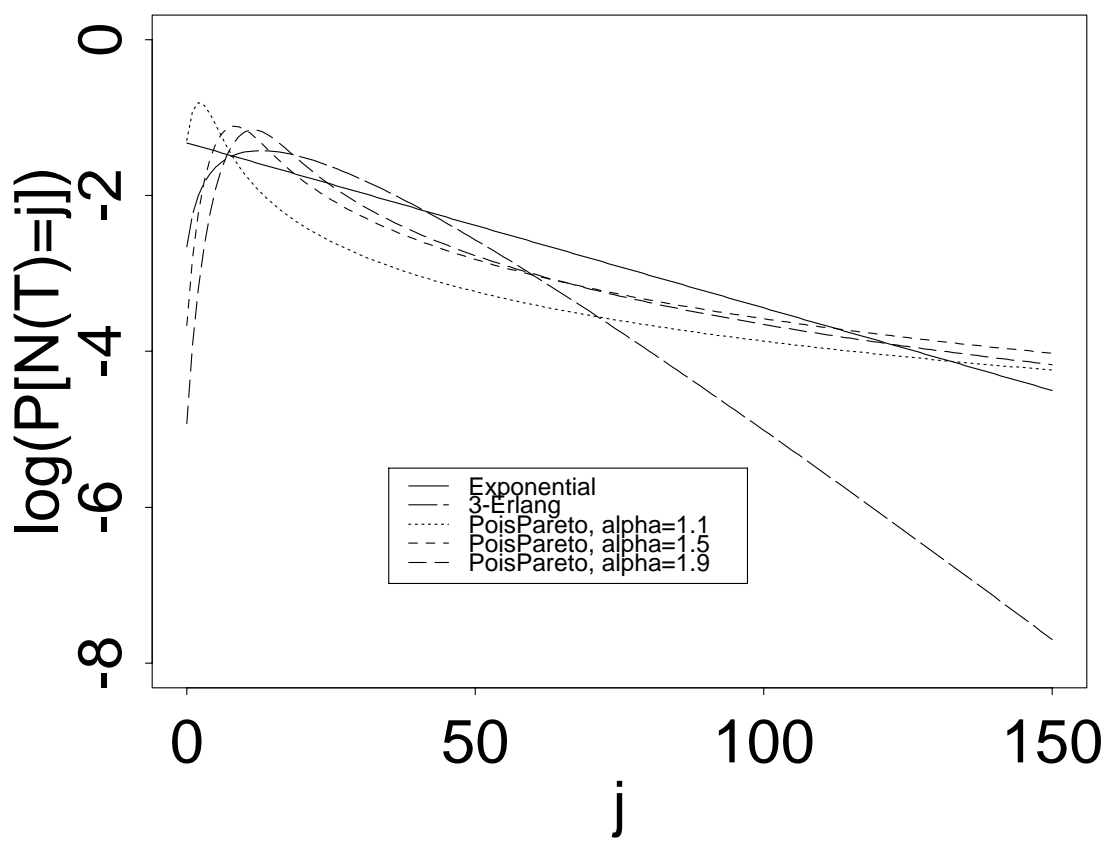

Figure 3: Logarithm of the pmf of the number of handovers, $\log _{10}(P[N(T)=j])$. We have fixed the mobility ratio as $\rho=20$, and we are comparing the following distributions for the CHT: Pareto with $\alpha=1.1,1.5$, and 1.9, 3-Erlang, and exponential. We assume the exponentially distributed CRT.

Thus we can confirm that $\mathbf{E}[N(T)]=\rho$, and the variance is given by

$$
\operatorname{Var}[N(T)]=\frac{\rho(1+\rho)\left(1+2 \rho+2 \rho^{2}\right)}{(1+2 \rho)^{2}} .
$$

We should mention that even though (88) is a mixture of two negative binomial distributions, the resulting $p m f$ is unimodal. A plot of this $p m f$ for several values of $\rho$ is shown in Figure 6. It is clear that the decay is slower when $\rho$ is larger.

In order to examine the dependence of the variance of $N(T)$ on the distributions of CHT and CRT, we compare several combinations numerically. To do so, let us use the following notation in Figure 7: EG means the exponentially distributed CHT and gamma distributed CRT; EE means both exponentially distributed CHT and CRT; $k$ ErE means the $k$-Erlang CHT and exponentially distributed CRT; and 2Er2Er means both 2-Erlang CHT and CRT. From left to right in Figure 7, the first curve corresponds to EG with $\alpha=0.1$, which shows the highest variance that increases as $\rho$ is increased. The second curve corresponds to EG with $\alpha=0.5$, and the third to EE. We observe that all the cases for EG with $\alpha<1$ have the higher variance than EE. The fourth and fifth curves correspond to EG with $\alpha=1.5$ and 3, respectively, for which the variance is no further reduced by increasing $\alpha$. Significant reduction in the variance is attained in the $k \mathrm{ErE}$ cases shown by the sixth $(2 \mathrm{ErE})$ and eighth $(8 \mathrm{ErE})$ curves, where the reduction is large 


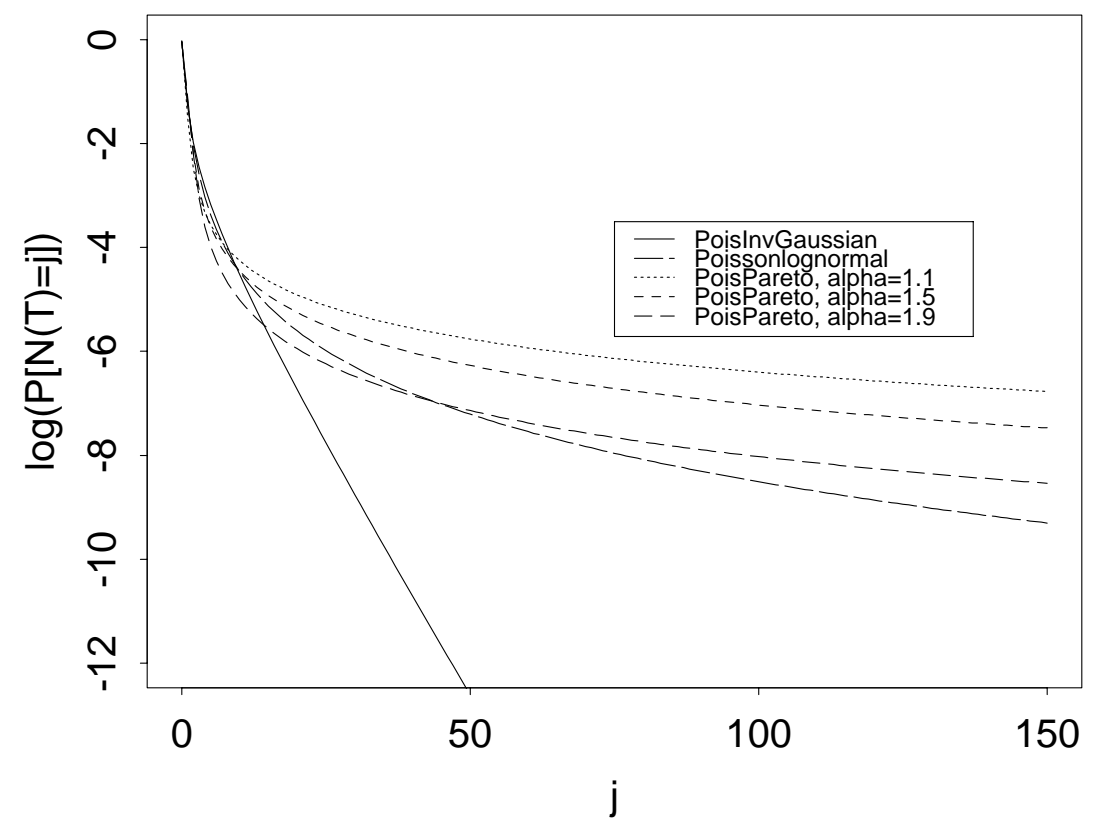

Figure 4: Logarithm of the pmf of the number of handovers, $\log _{10}(P[N(T)=j])$. We are comparing the Poisson-lognormal and the Poisson-Inverse Gaussian for mean 0.1 and variance 0.2 . We are also comparing the Poisson-Pareto with mean 0.1 and $\alpha=1.1,1.5$, and 1.9. We assume the exponentially distributed CRT.

when $k$ is large (the CHT has a shorter tail). On the other hand, the seventh curve for $2 \mathrm{Er} 2 \mathrm{Er}$ is only slightly lower than the sixth curve for $2 \mathrm{ErE}$. Thus it seems that changing the parameters in the CHT distribution has more important effect on the variance of $N(T)$ than changing the CRT parameters.

\section{General Expression for the Variance}

In Section 2, we have shown that $\mathbf{E}[N(T)]=\mathbf{E}[T] / \mathbf{E}[X]$ for the equilibrium renewal process in (8). In this section, we derive

$$
\operatorname{Var}[N(T)]=\int_{t=0}^{\infty} \operatorname{Var}[N(T) \mid T=t] f_{T}(t) d t+\frac{\operatorname{Var}[T]}{\mathbf{E}^{2}[X]} .
$$

To do so, we begin with the formula

$$
\operatorname{Var}[N(T)]=G_{N(T)}^{\prime \prime}(1)+G_{N(T)}^{\prime}(1)\left[1-G_{N(T)}^{\prime}(1)\right],
$$

where, from (2), we have

$$
G_{N(T)}^{\prime \prime}(1)=\left.\int_{t=0}^{\infty} \frac{d^{2}}{d z^{2}} G_{N(t)}(t, z)\right|_{z=1} f_{T}(t) d t
$$




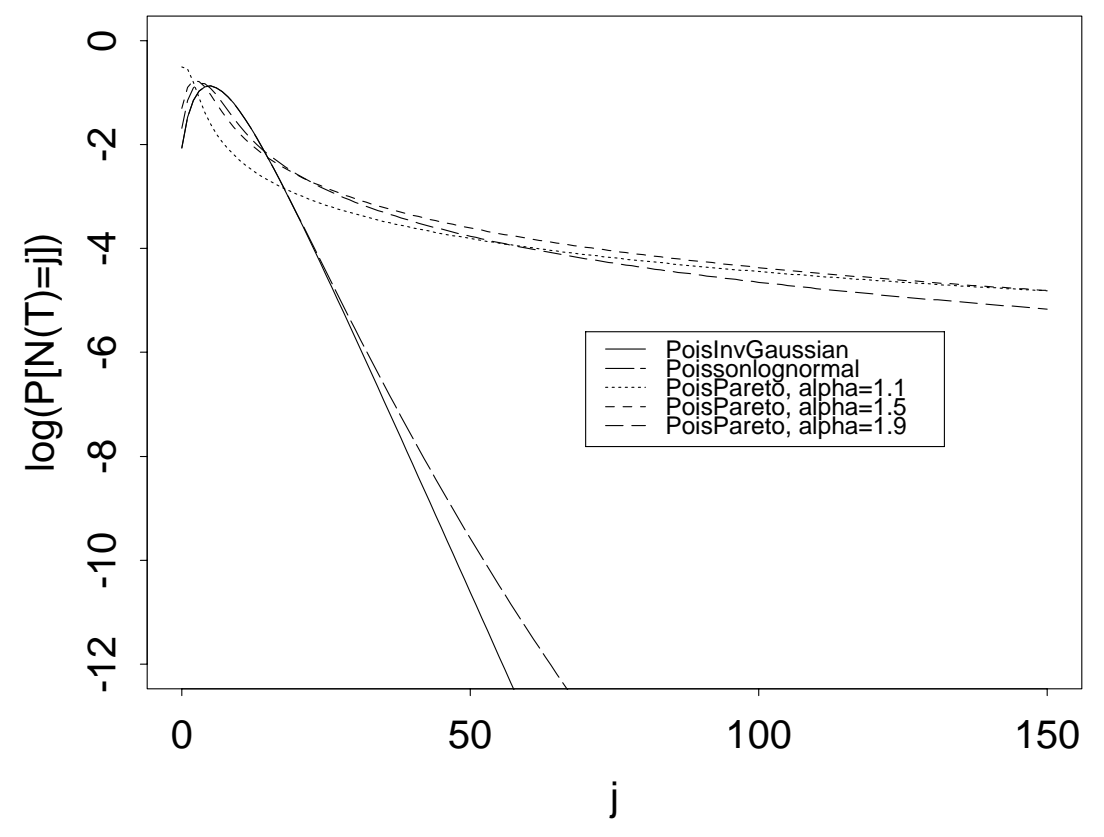

Figure 5: Logarithm of the pmf of the number of handovers, $\log _{10}(P[N(T)=j])$. We are comparing the Poisson lognormal and the Poisson Inverse Gaussian for mean 6 and variance 10. We are also comparing the Poisson-Pareto with mean 6 and $\alpha=1.1,1.5$, and 1.9. We assume the exponentially distributed CRT.

and

$$
\left.\frac{d^{2}}{d z^{2}} G_{N(t)}(t, z)\right|_{z=1}=\operatorname{Var}[N(T) \mid T=t]+\mathbf{E}^{2}[N(T) \mid T=t]-\mathbf{E}[N(T) \mid T=t] .
$$

Substituting (95) into (94) and then into (93), we get

$$
\begin{aligned}
\operatorname{Var}[N(T)] & =\int_{t=0}^{\infty} \operatorname{Var}[N(T) \mid T=t] f_{T}(t) d t \\
& +\int_{t=0}^{\infty} \mathbf{E}^{2}[N(T) \mid T=t] f_{T}(t) d t-\mathbf{E}^{2}[N(T)] .
\end{aligned}
$$

Equation (96) is valid not only for the equilibrium renewal process but also for the delayed renewal process. For the equilibrium renewal process, we have $\mathbf{E}[N(T) \mid T=t]=t / \mathbf{E}[X]$ and $\mathbf{E}[N(T)]=\mathbf{E}[T] / \mathbf{E}[X]$ as in (7) and (8), respectively. Hence we obtain (92).

Thus, if we have an explicit expression for the conditional variance $\operatorname{Var}[N(T) \mid T=t]$, we can apply (92) to obtain $\operatorname{Var}[N(T)]$. For instance, when the CRT is distributed as 2-Erlang $(\mu)$, the conditional variance has been obtained by Cox [3, p.58, eq.(12)] as

$$
\operatorname{Var}[N(T) \mid T=t]=\frac{\mu t}{4}+\frac{1}{8}-\frac{1}{8} e^{-2 \mu t} .
$$




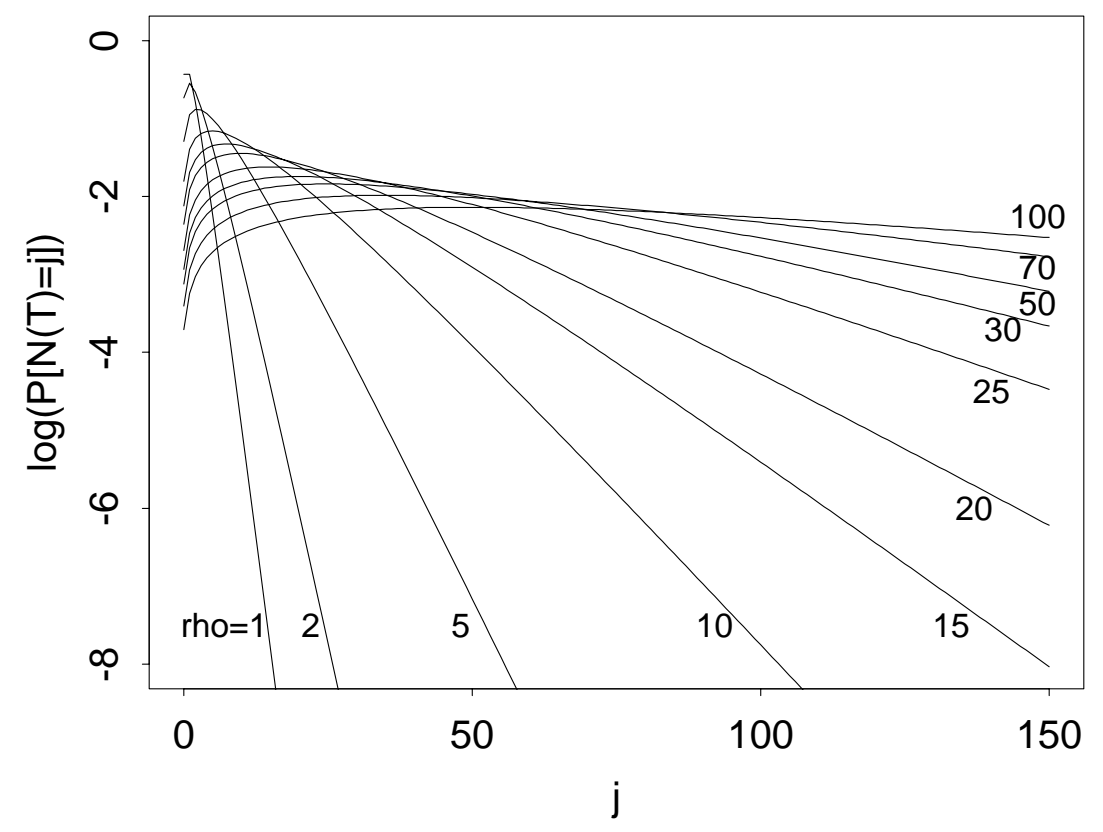

Figure 6: Logarithm of the pmf of the number of handovers, $\log _{10}(P[N(T)=j])$, for different values of $\rho$ when both CHT and CRT have 2-Erlang distributions.

Substituting this into (92) we get

$$
\operatorname{Var}[N(T)]=\frac{\mu}{4} \mathbf{E}[T]+\frac{1}{8}-\frac{1}{8} \int_{t=0}^{\infty} e^{-2 \mu t} f_{T}(t) d t+\frac{1}{4} \operatorname{Var}[T] \mu^{2} .
$$

By considering 2-Erlang $(\lambda)$ for the CHT, it is easy to recover (91).

\section{Conclusions}

In this paper, we have derived many explicit forms for the pmf and the statistical moments of the number of handovers during a random call holding time (CHT) when (a) the CHT distribution is well-fitted by a mixture of Erlang distributions while the CRT is arbitrarily distributed, and (b) the CRT is exponentially distributed while the CHT is arbitrary. The arbitrary distributions include the short-tailed Gamma distribution as well as the long-tailed lognormal, inverse Gaussian, and Pareto distributions. Many other interesting combinations of the CHT and CRT distributions can be handled in the same framework. Our method can be applicable to a variety of traffic situations in multimedia wireless networks with CHT whose coefficient of variation is larger or smaller than unity.

We should remark that obtaining the $p m f$ for the number of handovers during a call is an important step for obtaining other performance measures, including the probability 


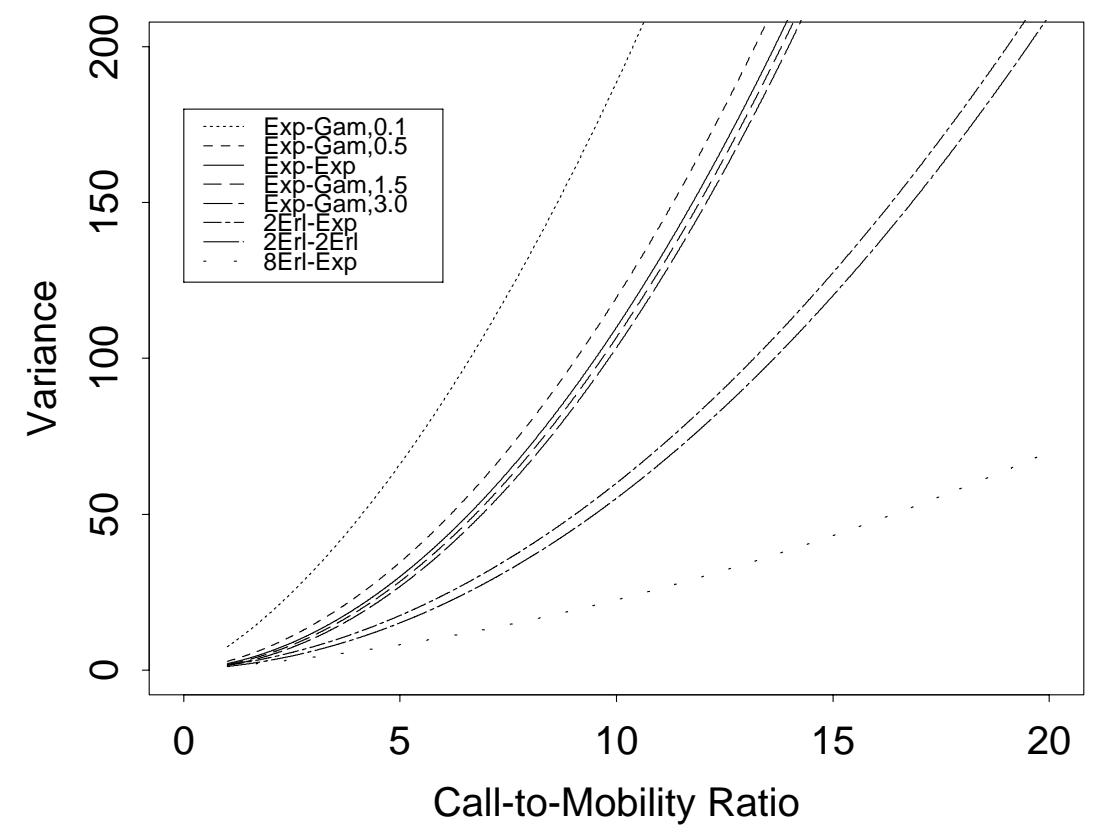

Figure 7: Variances of $N(T)$ for several distributions. From left to right, we have EG with $\alpha=0.1,0.5$; EE; EG with $\alpha=1.5,3$; 2ErE; 2Er2Er; and 8ErE.

of completing a call and the handover traffic rate, as it has been shown in $[16,17,19]$ as well as for designing the optimal mobility management algorithms [18].

We have proved that the mean number of handovers per call is always equal to the mobility ratio $\rho$, the ratio of the mean $\mathrm{CHT}$ to the mean CRT. We have also derived a general expression for the variance of the number of handovers. They are valid for any combination of the CHT and CRT distributions in the framework of the equilibrium renewal process.

\section{Acknowledgment}

The first author thanks the partial support provided by the Japan Society for the Promotion of Science (JSPS) and Conacyt for his visit to the University of Tsukuba.

\section{References}

[1] V. A. Bolotin, Y. Levy, and D. Liu, Characterizing data connection and messages by mixtures of distributions on logarithmic scale, ITC 16, P. Key and D. Smith (editors), pp.887-894. 1999. 
[2] E. Casilari, F. J. González, and F. Sandoval, Modeling of HTTP traffic, IEEE Communications Letters, Vol.5, No.6, pp.272-274, 2001.

[3] D. R. Cox, Renewal Theory, Methuen \& Co., London, 1962.

[4] M. E. Crovella and A. Bestavros, Self-similarity in world wide web traffic: Evidence and possible causes, IEEE/ACM Transactions on Networking, Vol.5, No.6, pp.835846, December 1997.

[5] Y. Fang, I. Chlamtac, and Y.-B. Lin, Call performance for a PCS network, IEEE Journal on Selected Areas in Communications, Vol.15, No.8, pp.1568-1581, October 1997.

[6] Y. Fang, I. Chlamtac, and Y.-B. Lin, Channel occupancy times and handoff rate for mobile computing and PCS networks, IEEE Transactions on Computers, Vol.47, No.6, pp.679-692, June 1998.

[7] I. S. Gradshteyn and I. M. Ryzhik, Table of Integrals, Series, and Products, 4th edn. Translated from the Russian by Scripta Technica, Academic Press, 1992.

[8] M. S. Holla, On a Poisson-inverse Gaussian distribution, Metrika, Vol.11, No.2, pp.115-121, 1966.

[9] L. Kleinrock, Queueing Systems, Volume 1: Theory, John Wiley \& Sons, New York, 1975 .

[10] Y.-B. Lin, S. Mohan, and A. Noerpel, Queueing priority channel assignment strategies for handoff and initial access for a PCS network, IEEE Transactions on Vehicular Technology, Vol.43, No.3, pp.704-712, 1994.

[11] Y.-B. Lin, Performance modeling for mobile telephone networks, IEEE Network Magazine, Vol.11, No.6, pp.63-68, 1997.

[12] Y.-B. Lin, Impact of PCS handoff response time, IEEE Communications Letters, Vol.1, No.6, pp.160-162, 1997.

[13] Y.-B. Lin, Modeling techniques for large-scale PCS networks, IEEE Communications Magazine, Vol.35, No.2, pp.102-107, February 1997.

[14] S. Nanda, Teletraffic models for urban and suburban microcells: Cell sizes and handhoff rates, IEEE Transactions on Vehicular Technology, Vol.42, No.4, pp.673682, November 1993.

[15] R. M. Rodríguez-Dagnino, Handoff analysis in wireless multimedia networks, SPIE Conference on Performance and Control of Network Systems II, Vol.3530, pp.76-84, 1998.

[16] R. M. Rodríguez-Dagnino, G. Hernández-Lozano, and H. Takagi, Wireless handover distributions in mixed platforms with multimedia services, SPIE Conference on Internet Quality and Performance and Control of Network Systems, Vol.4211, pp.59-69, 2000. 
[17] R. M. Rodríguez-Dagnino and C. A. Leyva-Valenzuela, Performance analysis in broadband wireless networks, SPIE Conference on Performance and Control of Network Systems III, Vol.3841, pp.220-228, 1999.

[18] R. M. Rodríguez-Dagnino, J. J. Ruiz-Cedillo, and H. Takagi, Dynamic mobility management for cellular networks: A delayed renewal process approach, Submitted to IEICE Transactions on Communications, 2001.

[19] R. M. Rodríguez-Dagnino and H. Takagi, Handover distributions for wireless cellular networks with Pareto call holding times, SPIE, ITCom 2001 Conference on Internet Performance and Control of Network Systems II, Vol.4523, 2001.

[20] S. A. Shaban, Poisson-lognormal distributions, Lognormal Distributions: Theory and Applications, E. L. Crow and K. Shimizu (editors), pp.195-210, Marcel Dekker, New York, 1988.

[21] E. T. Whittaker and G. N. Watson, A Course of Modern Analysis, Fourth edn., Cambridge University Press, London, 1999 (reprinted).

[22] M. M. Zonoozi and P. Dassanayake, User mobility modeling and characterization of mobility patterns, IEEE Journal on Selected Areas in Communications Vol.15, No.7, pp.1239-1252, September 1997. 